\title{
The Sixth International Conference on Urban Health Corresponding Author Index
}

Acosta, Henry, 11-07

Adhikari, Ram Saran, 01-13, 04-05

Agarwal, Siddarth, 01-03, 05-09, 09-08

Ahmad, Rafiush-shan, P15-01

Allaci, MaryAnn Sorensen, 04-10

Alt, Patricia, P15-04

Amaeshi, Ijeoma, P1-06

Arora, Daksha, 08-09

Ash, Veron, 02-05

Azodo, Clement, 08-18

Bailey, Mike, 03-07, 09-15

Barton, Heather, 08-19

Batra, Surabhi, 01-07, P1-07

Bayoumi, Ahmed, P8-03

Bazant, Eva S.,12-04

Beard, John, 04-06

Boateng, John, 05-07

Bokhour, Yvonne, 15-05

Borotkanics, Robert, P6-01

Bowie, J., 09-04

Brown, Bernadine, 11-08

Browne, Mario, P3-05

Burke, Jessica, 01-08, 04-07

Butler, James, 04-24, P16-01

Cadot, Emmanuelle, 04-11

Calikoglu, Sule, 18-01

Campbell, Jacquelyn, P12-01

Cardona, Kathleen, P18-02

Cardoso, Clareci Silva, 08-02, 08-03

Chaaya, M., 05-05

Charafeddine, Rana, P3-01

Chisolm, Deena, 01-02, P1-08

Cohen, Marcia, 01-11

Cone, James, 07-01

Cowdery, Joan, P7-01

Crilly, John, 03-10

Crisostomo, Josephine, 04-04

Cusimano, M., 16-02, P14-01

Dode, Aditza, 07-07

Dogra, N.K., 07-06

Domingos, Mirian Pereira, P5-02

Donde, Medha, 10-06

Dorsey, Rashida, P15-03

Dos Santos Diniz, Michelle, 01-09

Droppers, Oliver, 03-01

Easterbrook, J.D., P5-03

Eldberg, Mark, 10-08

Ellison, Gary, 04-22

Elmi, Ahmed, 10-07

Elmore, Shekinah, P13-01

Espinel, Eduardo, 12-02
Facente, Shelley N., 08-12

Fahs, Marianne, 04-02

Felix, Maria Leny, 08-13

Finkelstein, Joseph, 08-05

Foster-Drain, Robin, P2-03

Fotso, Jean-Christophe, 01-10, 04-18

Fraser, Alicia, 07-02

Frye, Victoria, 05-03

Fryer, Craig S., P16-04

Fuhrel, Andrea, 08-06

Gale, Sara, 03-04, 16-03

Garfein, Richard, 06-02

Garnes, Petra, P17-02

Genberg, Becky, 11-01

Gershon, Robyn, 09-05, 15-03, 15-04

Ghimire, Nirajan, 06-08, P18-01

Giri, Minal, 02-11

Gitomer, Shira, 14-02

Glazier, Rick, 04-25

Gluth, Dale, 09-13, P2-02

Goldman, Leslie, 01-04, 08-14, P16-03

Goodman, Melody, P4-03

Goodman, Jewel, P8-01

Guirguis-Younger, Manal, P10-03

Guy, Suzanne, 13-04

Habib, Rima, P6-02

Hajjar, Souraya, P11-01

Haldar, Partha, 08-10

Harbour, Catherine, 01-17, 10-10

Harvey, I. Shevon, 09-14

Hassan, Zeinab, 06-07

Heaphy, E.L.G., P5-04

Hernandez-Arias, P. Rafael, 10-04

Holding, Penny, P1-03

Hwang, Stephen, P12-03

Innis, Sarah, 04-12

Irish-Hauser, Sonya, 08-01

Jackson, Rachael, 05-08

Janjua, Naveed Zafar, 02-07

John, E.S., 12-05

Johnson, Daniel, 03-03

Kaufman, J.S., 05-01

Kaufman, Adriana, 02-03

Kebaso, John, 09-07

Khillare, Kishor, 06-03, P15-02

Khillare, Asha K., P12-02

Kidd, Sean, 11-03

Kirst, Maritt, 11-14
Klusanitz, Heather, 16-01

Kogon, M., P5-01

Komeh-Nkrumah, Steve, P3-04

Krishnapillai, A.D.S., 12-01

Kristjansson, Elizabeth, 04-23, 04-26

Kub, Joan, 04-01

Kumi-Kyereme, Akwasi, 01-14

Kuo, Irene, 11-09

Kwon, Simona, 13-02

Lapitan, Jostacio Moreno, 15-02

Lapshin, Oleg, 03-11

Lee, B.K., 05-13

Levy, Jessica, P4-06

Lewis-Land, Cassia, P1-05

Magnus, Manya, 06-09

Maharjan, Sabin, 02-01

Marshall, Brandon, 05-02

Marvis, Kodjovi, 11-02, 11-06, P9-01

Matheson, Flora, 11-13

Mawani, Farah, 10-02

Mercado, Joanny, P12-04

Messer, L.C., 07-03, P4-02

Metraux, Stephen, P10-05

Metzger, Kristina, 15-01

Miah, Golam Mothabbir, 02-10

Milloy, M.-J.S., 04-14

Milstein, Rachel, 04-27

Mino, Milton, 11-12

Minsky, Sara, 08-17

Mishra, S.I., 09-12

Mitchell, S. Gwin, 11-05

Moses, Marina, P3-02

Mudege, Netsayi Noris, 05-12

Munodawafa, Davison, 09-11, 09-18

Murray, Kantahyanee, P1-02

Nanda, Joy, 08-11

Nandi, Arjit, 05-11

Narimah, Adlina, 17-03

Naseem, Afshan, P4-05

Nnabuchi, Akpeh, 17-01

Norella, Loyd Brendan, 06-01

Nwaoha, F.O., 06-04

Oh, April, 04-17

Okonkwo, Kingsley, 06-06

OlaOlorun, F.M., 17-02

Oldham, Michael, 04-21

Olufayo, Olu-Olu, P6-03, P17-01

Ompad, Danielle, 04-20, 14-01

Omuemu, Vivian, P1-01

Otaj, Jane, 12-03

Palepu, Anita, 03-02 
Parenteau, M.-P., 03-05

Parker, Candace, 05-06

Passman, Dina, 01-15

Passos, Vitor, P4-09

Patierno, Steven, 18-03

Patterson, Tracy, 01-01, 01-12

Peterson, James, P4-10

Philbin, Morgan, 13-05

Pierotti, Deirdre, 02-08

Pleasant, Andrew, 05-10

Pollini, Robin, 11-04

Popiel, David, P1-04

Prasad, Korlagunta K. Sayi, P4-01

Putnam, Sara, 06-05

Rajasekhar, M., 07-04

Ramin, B., 03-08

Rashid, Audil, 10-01

Ratnayake, Ruwan, P10-04

Redwood, Yanique, 09-10

Regenstein, Marsha, 10-05

Reis, Ana Guilhermina, P4-08, P8-04

Rice, Dana, 02-02

Ritchie, A., 04-09
Roberts, Darryl, 03-12

Rouver, Charles-Antoine, 18-02

Rubin, A., 11-10

Ryder, Priscilla, 04-13

Saha, Subir, 04-19

Saliba, Matilda, 17-04

Saluja, Saurabh, 09-01

Sample, Hilary, P5-05

Schaefer, Jodi, P3-06

Schaefer-McDaniel, Nicole, 04-15

Schempf, Ashley, 04-16

Schootman, Mario, 04-03

Scott, A.N., P3-03

Scott, Rosalyn, 09-16

Scott, Edward, 08-20

Semogas, Dyanne, 08-08

Shaheen, Magda, 08-15, P7-02

Shanahan, C.W., 08-16

Shapcott, Michael, 02-09

Shelley, K.D., P4-07

Sison, Erica, 10-09

Srivastaya, Karishma, 09-02

Stephenson, Robert, 11-11

Steward, John, 02-06
Stillman, Frances, 09-06

Strathdee, S.A., P10-02

Suleiman, A., P8-05

Swahn, Monica, 01-16

Swanson, Karen, P10-01

Talnan, Edouard, 17-05

Thomas, Tammy, 01-06

Urquia, Marcelo, 10-03

Vaillancourt, Valine, P2-01

Van Brussel, Giel, 02-04

Vanguri, Poornima, 09-09

Warren, Jennifer, 08-04

Wessler, Sarah, 03-09

White, Heather, 04-08

Williams, Jessica, 01-05

Williams, C.T., 03-06

Wilson, Sacoby, 07-05

Yonas, Michael, 09-17, 13-01

Young, Heather, P4-04

Young, Pamela, 05-04

Young, Staci, P16-02

Younkin, S.L., 09-03

Younkin, Sharon, P8-02

Zarcadoolas, Christina, 08-07

Zenk, Shannon, 04-28, 13-03 